Series A

\author{
I. MATHEMATICA
}

310

\title{
GRUNSKY INEQUALITIES AND COEFFICIENTS OF BOUNDED SCHLICHT FUNCTIONS
}

VIKRAMADITYA SINGH 
Communicated 12 May 1961 by P.J. Myrberg and L. Ahlfors 


\section{Grunsky inequalities and coefficients of bounded schlicht functions*}

Let $D$ be a domain, having the origin as an interior point, in the complex $z$-plane winich is bounded by $n$ closed analytic curves $C_{v}$ and let $C=\sum_{v=1}^{n} C_{v}$ denote the boundary of $D$. Let $f(z)$ be a regular analytic function in $D$ which has the following expansion about the origin

$$
f(z)=b_{1} z+b_{2} z^{2}+b_{3} z^{3}+\ldots, \quad b_{1}>0 .
$$

Grunsky [5] had given a set of inequalities, depending upon the coefficients $b_{i}(i=1,2,3, \ldots)$ in (1), which are necessary and sufficient so that $f(z)$ may be extended to a schlicht function in the whole domain $D$. In the present paper we give the corresponding modification of the inequalities when $f(z)$ has an additional restriction of being bounded, i.e., $|f(z)|<1$, $z \in D$. Schiffer and Spencer [11] gave a generalization of Grunsky inequalities when $D$ is replaced by finite oriented Riemann surfaces and from there obtained the inequalities when $|f(z)|<1$. But their derivation in this special case does not seem to give sharp results.

We use the theory of Bergman kernel function and it is found that Grunsky inequalities are the outcome of a certain monotonicity result due to Bergman and Schiffer [2]. In the particular case when $D$ is the unit disc, $|z|<1$, we obtain the distortion theorems for the class $S$ of bounded schlicht functions in $|z|<1$ and using an observation by Charzynski and Schiffer [3] we obtain sharp bounds on $\left|b_{4}\right|$ when the coefficients $b_{n}$ are real.

The Grunsky inequalities are equally well adapted to the class $\Sigma$ of schlicht functions $\varphi(z)$ in $|z|>1$ such that $|\varphi(z)|>1$ in $|z|>1$, and

$$
\varphi(z)=c z+\frac{c_{1}}{z}+\frac{c_{2}}{z^{2}}+\frac{c_{3}}{z^{3}}+\ldots, \quad c>1,
$$

about the point at infinity. We have given bounds on $\left|c_{1}\right|$ and $\left|c_{2}\right|$ which include, as special cases, the inequalities due to Schiffer [8] and Golusin [4] for the class $\Sigma_{1}$ of functions $\psi(z)$ schlicht in $|z|>1$ and having the expansion

* This work was supported in part by the Air Force Office of Scientific Research, Air Research and Development Command, Washington 25, D.C., under Contract AF $49(638)-574$. 


$$
\psi(z)=z+\frac{c_{1}}{z}+\frac{c_{2}}{z^{2}}+\frac{c_{3}}{z^{3}}+\ldots
$$

We have also proved sharp inequalities for the first three coefficients of the class $\Sigma^{\prime}$ of functions which are inverse to functions of the class $\Sigma$, i.e., a function $g(w) \in \Sigma^{\prime}$ maps a schlicht domain containing $w=\infty$ and contained in $|w|>1$ onto $|z|>1$ and has the expansion

$$
g(w)=d w+\frac{d_{1}}{w}+\frac{d_{2}}{w^{2}}+\frac{d_{3}}{w^{3}}+\ldots .
$$

\section{§ 1. Grunsky Inequality}

Let $B$ be a bounded domain of connectivity $n$, in the $w$-plane, containing the origin and contained in $|w|<1$. Let the boundary $b$ of $B$ consist of $n$ closed analytic curves $b_{v}(v=1,2, \ldots, n)$ and let $b_{1}$ be the outer boundary and $\bar{B}_{v}$ the simply connected domain complementary to $B$ and bounded by $b_{\nu}$.

If the differential operators $\frac{\partial}{\partial w}$ and $\frac{\partial}{\partial \bar{w}}$ are defined by

$$
\frac{\partial}{\partial w}=\frac{1}{2}\left(\frac{\partial}{\partial u}-i \frac{\partial}{\partial v}\right), \frac{\partial}{\partial \bar{z}}=\frac{1}{2}\left(\frac{\partial}{\partial u}+i \frac{\partial}{\partial v}\right), w=u+i v,
$$

and $g(w, \zeta)$ is the Green's function of $B$, then it is well known [9] that

$$
K(w, \bar{\zeta})=-\frac{2}{\pi} \frac{\partial^{2} g(w, \zeta)}{\partial w \partial \bar{\zeta}},
$$

and

$$
L(w, \zeta)=\frac{1}{\pi(w-\zeta)^{2}}-\chi(w, \zeta)=-\frac{2}{\pi} \frac{\partial^{2} g(w, \zeta)}{\partial w \partial \zeta},
$$

where $K(w, \bar{\zeta})$ is the Bergman kernel function of $B$ and $\chi(w, \zeta)$ is regular analytic in the closed domain $B+b$ and is symmetric in $w$ and $\zeta$.

Consider the function

$$
p(w)=\operatorname{Re}\left\{\sum_{\nu=1}^{n} \alpha_{\nu} \frac{\partial g\left(w, \zeta_{\nu}\right)}{\partial \zeta_{\nu}}\right\},
$$

where $\alpha_{\nu}$ are arbitrary complex numbers and $\zeta_{v} \in B . p(w)$ is harmonic in $B$, except at $\zeta_{v}$, and vanishes on the boundary $b$. Its singular part is given by 


$$
s_{1}(w)=\frac{1}{2} \operatorname{Re}\left\{\sum_{\nu=1}^{n} \frac{1}{w-\zeta_{\nu}}\right\}
$$

Therefore, if

$$
S(w)=-\frac{1}{2} \operatorname{Re}\left\{\sum_{\nu=1}^{n}\left(\frac{\alpha_{v}}{w-\zeta_{\nu}}-\frac{\bar{\alpha}_{\nu} w}{1-\bar{\zeta}_{\nu} w}\right)\right\},
$$

the function $p(w)+S(w)$ is harmonic in $B$ and by Green's theorem

$$
\int_{b}(p(w)+S(w)) \frac{\partial}{\partial \tilde{n}}(p(w)+S(w)) d s \geq 0
$$

where $\tilde{n}$ is the outer normal with respect to $B$. Since $p(w)=0, w \in b,(10)$ yields

$$
\int_{b} S(w) \frac{\partial p(w)}{\partial \tilde{n}} d s \geq-\int_{b} S(w) \frac{\partial S(w)}{\partial \tilde{n}} d s .
$$

In view of the fact that $S(w)$ is harmonic in $\bar{B}_{v}(v=2,3, \ldots, n)$ we obtain by virtue of Green's theorem

$$
\begin{aligned}
& -\int_{b} S(w) \frac{\partial S(w)}{\partial \tilde{n}} d s \\
& =\frac{1}{4} \sum_{\mu=2}^{n} \int_{\bar{B}_{\mu}} \int_{\mu-v=1} \sum_{\left(w-\zeta_{\nu}\right)^{2}}^{n}\left(\frac{\bar{\alpha}_{v}}{\left(w-\tau_{w}-\int_{b_{1}} S(w) \frac{\partial S(w)}{\partial \tilde{n}} d s .\right.}\right.
\end{aligned}
$$

In order to see that the last term in (12) is also non-negative we consider the region $E$ or the union of finite number of regions $E_{v}$, bounded by the unit circumference and $b_{1}$. Then $S(w)$ is harmonic in this and vanishes on $|w|=1$. Hence

$$
-\int_{b_{1}} S(w) \frac{\partial S(w)}{\partial \tilde{n}} d s=-\int_{b_{1}+|w|=1} S(w) \frac{\partial S(w)}{\partial \tilde{n}} d s \geq 0,
$$

by Green's theorem. Equality in (13) occurs only when $E$ or $E_{v}$ have zero area and hence $b_{1}$ contains $|w|=1$.

The expression on the left hand side of (11) can be integrated explicitly by the residue theorem. Indeed, if $U(w)$ and $V(w)$ are analytic functions for which $p(w)=\operatorname{Re}(U(w))$ and $S(w)=\operatorname{Re}\{V(w)\}$, we have, in view of $p(w)=0, w \in b$,

$$
\frac{\partial p(w)}{\partial \tilde{n}} d s=\frac{1}{i} U^{\prime}(w) d w, \quad w \in b
$$


A. I. 310

Hence

$$
\begin{aligned}
\int_{b} S(w) \frac{\partial p(w)}{\partial \tilde{n}} d s & =\int_{b}(p(w)+S(w)) \frac{\partial p(w)}{\partial \tilde{n}} d s \\
& =\operatorname{Re}\left\{\frac{1}{i} \int_{b}(U(w)+V(w)) U^{\prime}(w) d w\right\} \\
& =-\operatorname{Re}\left\{\frac{1}{i} \int_{b}(U(w)+V(w)) V^{\prime}(w) d w\right\},
\end{aligned}
$$

use having been made of the fact that $U(w)+V(w)$ is regular in $B$. To evaluate the above integral we observe that

$$
U(w)=\frac{1}{2} \sum_{\nu=1}^{n}\left[\alpha_{\nu} \frac{\partial q\left(w, \zeta_{\nu}\right)}{\partial \zeta_{\nu}}+\bar{\alpha}_{\nu} \frac{\partial q\left(u, \zeta_{\nu}\right)}{\partial \bar{\zeta}_{\nu}}\right]
$$

where $q(w, \zeta)$ is the analytic function in $w$ for which $\operatorname{Re}\{q(w, \zeta)\}=g(w, \zeta)$. Also, it is easily verified that

$$
\frac{\partial q(w, \zeta)}{\partial w}=2 \frac{\partial g(w, \zeta)}{\partial w}
$$

Therefore, in view of the relations (5), (6), and (15) we obtain from (14) (16)

$$
\begin{aligned}
& \int_{b} S(w) \frac{\partial p(w)}{\partial \tilde{n}} d s \\
& =\pi^{2} \operatorname{Re}\left\{\sum_{\nu, \mu=1}^{n} \alpha_{\nu} \bar{\alpha}_{\mu}\left[K\left(\zeta_{\nu}, \bar{\zeta}_{\mu}\right)-\frac{1}{\pi\left(1-\zeta_{\nu} \bar{\zeta}_{\mu}\right)^{2}}\right]-\sum_{\nu=1}^{n} \alpha_{\nu} \alpha_{\mu} \chi\left(\zeta_{\nu}, \zeta_{\mu}\right)\right\} .
\end{aligned}
$$

Hence (11), (12), (13), and (16) yield

$$
\operatorname{Re}\left\{\sum_{\nu, \mu=1}^{n} \alpha_{\nu} \bar{\alpha}_{\mu}\left[K\left(\zeta_{\nu}, \bar{\zeta}_{\mu}\right)-\frac{1}{\pi\left(1-\zeta_{v} \bar{\zeta}_{\mu}\right)^{2}}\right]-\sum_{\nu, \mu=1}^{n} \alpha_{\nu} \alpha_{\mu} \gamma_{\nu}\left(\zeta_{\nu}, \zeta_{\mu}\right)\right\} \geq 0 .
$$

Equality in (17) is attained only when equality holds in (10) and the right hand side of (11) vanishes. The former equality will hold if $p(w)+S(w)$ is constant in $B$ or equivalently on $b$. Since $p(w)=0, w \in b$, this implies that the boundary $b$ should be given by

$$
\operatorname{Re}\left\{\sum_{\nu=1}^{n}\left[\frac{\alpha_{v}}{w-\zeta_{\nu}}-\frac{\bar{a}_{\nu} w}{1-\bar{\zeta}_{v} w}\right]\right\}=\text { const. }
$$


Further, from (12) and (13) we infer that the right hand side of (11) vanishes if the domains $\bar{B}_{v}$ contained in $|w|<1$ have no interior point and $b_{1}$ contains $|w|=1$. Since the constant in the right hand side of (18) vanishes if $|w|=1$ we conclude that the boundary of $B$ is given by

$$
\operatorname{Re}\left\{\sum_{\nu=1}^{n}\left[\frac{\alpha_{v}}{w-\zeta_{v}}-\frac{\bar{\alpha}_{v} w}{1-\bar{\zeta}_{v} w}\right]\right\}=0 .
$$

We note that (17) can equivalently be written in the form

$$
\left|\sum_{\nu, \mu=1}^{n} \alpha_{\nu} \alpha_{\mu} \chi\left(\zeta_{\mu}, \zeta_{\nu}\right)\right| \leq \sum_{\mu, \nu=1}^{n} \alpha_{\nu} \bar{\alpha}_{\mu}\left[K\left(\zeta_{\nu}, \bar{\zeta}_{\mu}\right)-\frac{1}{\pi\left(1-\zeta_{\nu} \bar{\zeta}_{\mu}\right)^{2}}\right]
$$

because if we replace $\alpha_{v}$ by $\alpha_{v} e^{i \Theta}, \Theta$ real and arbitrary, the part involving $\alpha_{\nu} \bar{\alpha}_{\mu}$ remains unchanged but the $\operatorname{Re}\left\{\sum_{\mu, v=1}^{n} \alpha_{\mu} \alpha_{\nu} \chi\left(\zeta_{\nu}, \zeta_{\mu}\right)\right\}$ gets transformed to $\operatorname{Re}\left\{e^{2 i \Theta} \sum \alpha_{\mu} \alpha_{\nu} \chi\left(\zeta_{\mu}, \zeta_{\nu}\right)\right\}$ where $\Theta$ can be so chosen that the real part becomes equal to the modulus. Further, we had assumed the boundaries to be analytic curves and we find that the extremal domains being slit domains do not belong to this class. But this can easily be avoided by using the continuity of $K(z, \bar{\zeta})$ and $\chi(z, \zeta)$ in dependence of their domain of definition $B$ and the preceding inequalities consequently hold for the most general domains.

Let $D$ be a domain in the $z$-plane as described above and let $w=f(z)$ map $D$ conformally onto the domain $B$. The relations (5) and (6) together with the conformal invariance of the Green's function readily yield the following transformation properties

$$
\begin{aligned}
& K(z, \bar{\zeta})=K\left(w, \zeta_{1}\right) f^{\prime}(z) \overline{f^{\prime}(\zeta)}, \quad \zeta_{1}=f(\zeta) \\
& \chi(z, \zeta)=\chi\left(w, \zeta_{1}\right) f^{\prime}(z) f^{\prime}(\zeta)+U(z, \zeta),
\end{aligned}
$$

where

$$
U(z, \zeta)=\frac{1}{\pi}\left\{\frac{f^{\prime}(z) f^{\prime}(\zeta)}{(f(z)-f(\zeta))^{2}}-\frac{1}{(z-\zeta)^{2}}\right\}=\frac{1}{\pi} \frac{\partial^{2}}{\partial z \partial \zeta} \log \frac{f(z)-f(\zeta)}{z-\zeta} .
$$

We have, therefore, demonstrated the following

Theorem 1. Let $D$ be a finitely connected plane domain and let $\eta_{\nu} \in D$. If $w=f(z)$ maps $D$ conformally onto a domain $B$ contained in $|w|<1$, and $\alpha_{v}$ are arbitrary complex numbers, then

$$
\begin{aligned}
& \left|\sum_{\nu, \mu=1} \alpha_{v} \alpha_{\mu}\left[\chi\left(\eta_{v}, \eta_{\mu}\right)+U\left(\eta_{v}, \eta_{\mu}\right)\right]\right| \\
& \leq \sum \alpha_{\nu} \bar{\alpha}_{\mu}\left[K\left(\eta_{\nu}, \bar{\eta}_{\mu}\right)-\frac{f^{\prime}\left(\eta_{\nu}\right) \overline{f^{\prime}\left(\eta_{\mu}\right)}}{\pi\left(1-f\left(\eta_{\nu}\right) \overline{f\left(\eta_{\mu}\right)}\right)^{2}}\right]
\end{aligned}
$$


where $K(z, \bar{\zeta})$ is the Bergman kernel of $D$ and $\chi(z, \zeta)$ and $U(z, \zeta)$ are defined by (6) and (23) respectively. For a given set of $\alpha_{v}$ some inequality becomes an equality if the image of $D$ under $f(z)$ has no exterior points in $|w|<1$ and its boundary satisfies the equation (19).

We remark that a partial converse of the above theorem is also true, namely, if $D$ is mapped on a slit subdomain $B$ of $|w|<1$ such that the boundary consists of the unit circumference together with slits which satisfy (19); then the mapping solves some extremum problem for the inequalities (24). To see this, consider

$R(w)=\sum_{\nu=1}^{n} \alpha_{\nu}\left(L\left(w, \zeta_{\nu}\right)-\frac{1}{\pi\left(w-\zeta_{\nu}\right)^{2}}\right)+\sum_{\nu=1}^{n} \bar{\alpha}_{\nu}\left(K\left(w, \bar{\zeta}_{\nu}\right)-\frac{1}{\pi\left(1-w \bar{\zeta}_{\nu}\right)^{2}}\right)$.

This represents a regular analytic function in $B$ and for $w \in b$, in view of (19) and the boundary relation

$$
L\left(w, \zeta_{v}\right) d w=-\overline{K\left(w, \overline{\zeta_{v}}\right)} \overline{d w},
$$

we have

$$
\operatorname{Re}(R(w) d w)=0 .
$$

Hence $R(w)$ vanishes identically and we obtain from (25)

$$
-\sum_{\nu=1}^{n} \alpha_{\nu} \chi\left(w, \zeta_{\nu}\right)+\sum_{\nu=1}^{n} \bar{\alpha}_{\nu}\left(K\left(w, \bar{\zeta}_{\nu}\right)-\frac{1}{\pi\left(1-w \bar{\zeta}_{\nu}\right)^{2}}\right)=0 .
$$

Taking norm over $B$ of (28) we obtain

$$
\begin{aligned}
\left\|\sum_{\nu=1}^{n} \alpha_{\nu} \chi\left(w, \zeta_{v}\right)\right\|^{2} & -2 \operatorname{Re} \int_{\mu, v=1}^{n} \sum_{\mu} \alpha_{v}\left(\chi\left(\zeta_{\mu}, \zeta_{v}\right)-\frac{1}{\tau} \iint \frac{\chi\left(w, \zeta_{\nu}\right)}{\left(1-\bar{w} \zeta_{u}\right)^{2}} d \tau_{w}\right) ! \\
& +\| \sum_{\nu=1}^{n} \bar{\alpha}_{\nu}\left(K\left(w, \bar{\zeta}_{v}\right)-\left.\frac{1}{\pi\left(1-w \overline{\zeta_{\nu}}\right)^{2}}\right|^{2}=0 .\right.
\end{aligned}
$$

Further, since the norm of both terms in (28) should be separately equal we get from (29)

$$
\left\|\sum_{\nu=1}^{n} \alpha_{v} \chi\left(w, \zeta_{\nu}\right)\right\|^{2}=\operatorname{Re}\left\{\sum_{\mu, \nu=1}^{n} \alpha_{\mu} \alpha_{\nu}\left(\chi\left(\zeta_{\mu}, \zeta_{\nu}\right)-\frac{1}{\pi} \iint \frac{\chi\left(w, \zeta_{\nu}\right)}{\left(1-\bar{w} \zeta_{\mu}\right)^{2}} d \tau_{w}\right)\right\} .
$$


It is easy to verify that

$$
\left\|\sum_{\nu=1}^{n} \alpha_{\nu} \chi\left(w, \zeta_{v}\right)\right\|^{2}=\sum_{\mu, v=1}^{n} \alpha_{\mu} \bar{\alpha}_{\nu}\left(K\left(\zeta_{\mu}, \bar{\zeta}_{v}\right)-\Gamma\left(\zeta_{\mu}, \bar{\zeta}_{\nu}\right)\right)
$$

where

$$
\Gamma\left(\zeta_{\mu}, \bar{\zeta}_{v}\right)=\frac{1}{2 \pi^{2} i} \int_{b} \frac{d w}{\left(w-\zeta_{\mu}\right)^{2}\left(\bar{w}-\bar{\zeta}_{v}\right)} .
$$

On the boundary slits this integral is readily seen to vanish and, by residue theorem, it can be evaluated on the unit circumference. In the present case, it is found that

$$
\Gamma\left(\zeta_{\mu}, \bar{\zeta}_{\nu}\right)=\frac{1}{\pi\left(1-\zeta_{\mu} \bar{\zeta}_{\nu}\right)^{2}}
$$

Also, by virtue of Green's theorem and the boundary relation (26), we have

$$
\begin{aligned}
\frac{1}{\pi} \iint_{B} \frac{\chi\left(w, \zeta_{v}\right)}{\left(1-\bar{w} \zeta_{\mu}\right)^{2}} d \tau_{w} & =\frac{1}{2 \pi i} \int_{b} \chi\left(w, \zeta_{v}\right) \frac{d w \bar{w}}{\left(1-\zeta_{\mu} \bar{w}\right)} \\
& =\frac{1}{2 \pi^{2} i} \int_{b} \frac{\bar{w} d w}{\left(w-\zeta_{v}\right)^{2}\left(1-\zeta_{\mu} \bar{w}\right)} \\
& =0 .
\end{aligned}
$$

Vanishing of the last integral is again a consequence of the fact that it vanishes on the boundary slits and also on the unit circumference. Therefore, (30) yields

$$
\sum_{\mu, v=1}^{n} \alpha_{\mu} \bar{\alpha}_{\nu}\left(K\left(\zeta_{\mu}, \bar{\zeta}_{v}\right)-\frac{1}{\pi\left(1-\zeta_{\mu} \bar{\zeta}_{v}\right)^{2}}\right)=\operatorname{Re}\left\{\sum_{\mu, v=1}^{n} \alpha_{\mu} \alpha_{\nu} \chi\left(\zeta_{\mu}, \zeta_{\nu}\right)\right\}
$$

Comparing this with (20) we see that the extremal character of $B$ with respect to that inequality is established. Since $(24)$ is a direct consequence of $(20)$ this proves our assertion for the converse that every domain contained in $|w|<1$ and bounded by $|w|=1$ and the slits satisfying (19) results from an extremal mapping.

It is worth pointing out that the inequality (20) is an immediate consequence of the fact that the quantity

$$
\operatorname{Re}\left\{\sum_{\nu, \mu=1}^{n} \alpha_{\nu} \bar{\alpha}_{\mu} K\left(\zeta_{\nu}, \bar{\zeta}_{\mu}\right)-\sum_{\nu, \mu=1}^{n} \alpha_{\nu} \alpha_{\mu} \chi\left(\zeta_{\nu}, \zeta_{\mu}\right)\right\}
$$

decreases if $D$ increases, once it has been demonstrated that it is always non-negative. Indeed, if the domain is contained in the unit circle, then 


$$
\begin{aligned}
\operatorname{Re}\left\{\sum_{\nu, \mu=1}^{n} \alpha_{\nu} \bar{\alpha}_{\mu} K\left(\zeta_{\nu}, \bar{\zeta}_{\mu}\right)\right. & \left.-\sum_{\nu, \mu=1}^{n} \alpha_{v} \alpha_{\mu} \chi\left(\zeta_{\nu}, \zeta_{\mu}\right)\right\} \\
& \geq \sum_{\nu, \mu=1}^{n} \frac{\alpha_{\nu} \bar{\alpha}_{\mu}}{\pi\left(1-\zeta_{\nu} \zeta_{\mu}\right)^{2}}
\end{aligned}
$$

because $\chi(z, \zeta) \equiv 0$ for the circle and $K(z, \bar{\zeta})=\frac{1}{\pi(1-z \bar{\zeta})^{2}}$. The monotonic character of (35) was proved by Bergman and Schiffer [2] by a variational method and also follows from the right hand side of (11) as was pointed out by Nehari [6]. But the fact that the quantity (35) is always non-negative seems to have escaped the attention of the earlier investigators. This observation makes it possible to obtain analogues of (24) for any other subclasses of the classes of conformal mappings of $D$.

If in (24) we specialize to $n=1$, we obtain

$$
\left|\chi(z, z)-\frac{1}{6 \pi}\{f(z), z\}\right| \leq K(z, \bar{z})-\frac{1}{\pi} \frac{\left|f^{\prime}(z)\right|^{2}}{\left(1-|f(z)|^{2}\right)^{2}},
$$

where $\{f(z), z\}$ is the well-known Schwarzian and we have used the fact that

$$
U(z, z)=-\frac{1}{6 \tau}\{f(z), z\} .
$$

The inequality (37) gives a generalization to the case of bounded univalent functions in $D$ of the inequality

$$
\left|\chi(z, z)-\frac{1}{6 \pi}\{f(z), z\}\right| \leq K(z, \bar{z})
$$

for univalent functions in $D$ due to Bergman and Schiffer [2].

Let us suppose that the origin is contained in $D$ and let us expand the analytic functions occurring in (24) into the power series

$$
\begin{aligned}
& U(z, \zeta)=\frac{1}{\pi} \sum_{\mu, \nu=0}^{\infty} C_{\mu \nu} z^{u} \zeta^{v}, \quad C_{\mu \nu}=C_{\nu \mu}, \\
& \chi(z, \zeta)=\sum_{\mu, v=0}^{\infty} \chi_{\mu \nu} z^{u \zeta^{\nu}}, \quad \chi_{\mu \nu}=\chi_{\nu \mu}, \\
& K(z, \bar{\zeta})=\sum_{\mu, v=0}^{\infty} k_{\mu \nu} z^{u} \bar{\zeta}^{\nu}, \quad k_{\mu \nu}=\bar{k}_{\nu \mu}, \\
& \frac{f^{\prime}(z) \overline{f^{\prime}(\zeta)}}{\left(1-f(z) \overline{f(\zeta))^{2}}\right.}=\sum_{\mu, \nu=0}^{\infty} d_{\mu \nu} z^{u} \bar{\zeta}^{v}, \quad d_{\mu \nu}=\bar{d}_{\nu \mu} .
\end{aligned}
$$


We notice that only the coefficients $C_{\mu \nu}$ and $d_{\mu \nu}$ involve the coefficients $b_{n}$ of the functions $f(z)$ given by (1). We now proceed to prove the following generalization of Grunsky's inequalities.

Theorem 2. Let $f(z)$ be a single-valued regular function in a finitely connected domain $D$ containing the origin and having the expansion (1). In order that $f(z)$ be schlicht and $|f(z)|<1$ in $D$ it is necessary and sufficient that for every complex vector $\alpha_{0}, \ldots, \alpha_{N}$

$$
\begin{aligned}
\left|\sum_{\mu, \nu=0}^{N}\left(\chi_{\mu \nu}+\frac{1}{\pi} C_{\mu \nu}\right) \alpha_{\mu} \alpha_{\nu}\right| \leq \sum_{\mu, \nu=0}^{N}\left(k_{\mu \nu}-\frac{1}{\pi} d_{\mu \nu}\right) \alpha_{\mu} \bar{\alpha}_{\nu}, \\
N=0,1,2, \ldots,
\end{aligned}
$$

where the coefficients $C_{\mu \nu}, \chi_{\mu \nu}, k_{\mu \nu}$, and $d_{\mu \nu}$ are defined in (39). For a given set of $\alpha_{v}$ some inequality in $(40)$ becomes an equality for such a schlicht function which maps $D$ on a domain bounded by the unit circumference and slit along an isothermal system of algebraic curves.

Proof. Let us suppose that $f(z)$ is bounded and schlicht in $D$. It therefore satisfies the inequality (24). Let $\Sigma$ be a closed rectifiable curve surrounding the origin and contained in the common domain of convergence of the developments (39) and let $p(s)$ be a complex-valued function of the length parameter on $\Sigma$ given by $p(s)=q(z(s)) z^{\prime}(s)$ where

$$
q(z)=\frac{1}{2 \pi i} \sum_{v=0}^{N} \alpha_{\nu} z^{-(v+1)} .
$$

We then obtain from (24) by a limit process

$$
\begin{aligned}
& \mid \int_{z, \zeta \in \in_{\Sigma}}[\chi(z, \zeta)+U(z, \zeta)] p\left(s_{z}\right) p\left(s_{\zeta}\right) d s_{z} d s_{\zeta} \\
& \leq \int_{z, \zeta \in \in_{\Sigma}}\left[K(z, \bar{\zeta})-\frac{f^{\prime}(z) \overline{f^{\prime}(\zeta)}}{\pi(1-f(z) \overline{f(\zeta)})^{2}}\right] p\left(s_{z}\right) \overline{p\left(s_{\zeta}\right)} d s_{z} d s_{\zeta} .
\end{aligned}
$$

This readily yields the inequality (40) and demonstrates the necessity of the conditions.

We now show that if (40) holds for every positive integral $N$ and every choice of $\alpha_{\mu}$ then $f(z)$ is univalent and $|f(z)|<1$. The univalence of $f(z)$ can be proved on the same lines as Bergman and Schiffer [2]. Indeed, because $\sum_{\mu, \nu=0}^{N} d_{\mu \nu} \alpha_{\mu} \bar{\alpha}_{\nu}$ is a positive definite form, (40) implies a fortiori

$$
\left|\sum_{\mu, \nu=0}^{N}\left(\chi_{\mu \nu}+\frac{1}{\pi} C_{\mu \nu}\right) \alpha_{\mu} \alpha_{\nu}\right| \leq \sum_{\mu, \nu=0}^{N} k_{\mu \nu} \alpha_{\mu} \bar{\alpha}_{\nu}, \quad N=0,1,2, \ldots,
$$


which is the equivalent form for the original Grunsky inequalities and for which schlichtness is well known. It therefore remains to demonstrate that $|f(z)|<1$. This is a consequence of the fact that the right hand side of (40) is positive. Indeed, set $\alpha_{\mu}=z^{\mu}+\zeta^{\mu}, \mu=0,1, \ldots, N, \alpha_{\mu}=0, \mu<N$. It then follows from the positiveness of the right hand side of $(40)$ that

$$
\begin{gathered}
\pi[K(z, \bar{z})+K(\zeta, \bar{\zeta})+K(\zeta, \bar{z})+K(z, \bar{\zeta})] \\
\geq \frac{\left|f^{\prime}(z)\right|^{2}}{\left(1-|f(z)|^{2}\right)^{2}}+\frac{\left|f^{\prime}(\zeta)\right|^{2}}{\left(1-|f(\zeta)|^{2}\right)^{2}}+2 \operatorname{Re}\left\{\frac{f^{\prime}(z) \overline{f^{\prime}(\zeta)}}{(1-f(z) \overline{f(\zeta)})^{2}}\right\} .
\end{gathered}
$$

Letting $z \rightarrow \zeta$ we find that

$$
\pi K(\zeta, \bar{\zeta}) \geq \frac{\left|f^{\prime}(\zeta)\right|}{\left(1-|f(\zeta)|^{2}\right)^{2}} .
$$

Now the facts that $K(\zeta, \bar{\zeta})$ is bounded for $\zeta \in D$ and $f(z)$ is regular analytic in $D$ such that $f(0)=0$ ensure that $|f(\zeta)|<1$.

\section{§ 2. Bounded Univalent Functions in $|z|<1$}

Let us suppose that the domain $D$ is the unit disc $|z|<1$. Inequalities (24) then take the form

$$
\begin{aligned}
& \mid \sum_{\mu, \nu=1}^{N} \alpha_{\nu} \alpha_{\mu}\left\{\frac{f^{\prime}\left(\zeta_{\nu}\right) f^{\prime}\left(\zeta_{\mu}\right)}{\left(f\left(\zeta_{v}\right)-f\left(\zeta_{\mu}\right)\right)^{2}}-\frac{1}{\left(\zeta_{v}-\overline{\left.\zeta_{\mu}\right)^{2}} \mid\right.} \mid\right. \\
& \quad \leq \sum_{\mu, \nu=1}^{N} \alpha_{\mu} \bar{\alpha}_{v}\left[\frac{1}{\left(1-\zeta_{\mu} \bar{\zeta}_{v}\right)^{2}}-\frac{f^{\prime}\left(\zeta_{\mu}\right) \overline{f^{\prime}\left(\zeta_{v}\right)}}{\left(1-f\left(\zeta_{\mu}\right) \overline{f\left(\zeta_{v}\right)}\right)^{2}}\right] .
\end{aligned}
$$

A similar result but of a different form was obtained by Nehari [6].

We proceed to derive from (43) the distortion theorems for the class $S$ of bounded univalent functions in $|z|<1$. We first obtain distortion theorems when $f(z)$ is an odd univalent function, i.e., $f(z)=-f(-z)$. Let us put $N=2, \zeta_{1}=z, \zeta_{2}=-z$, and $\alpha_{1}=i \alpha_{2}$ in (43). We get

$$
\mid \frac{f^{\prime}\left(z^{2}\right)}{f^{2}(z)}-\frac{1}{z^{2}} \leq \frac{4}{\left(1-z^{2}\right)^{2}}-\frac{4\left|f^{\prime}(z)\right|^{2}}{\left(1-|f(z)|^{2}\right)^{2}}
$$

which yields

$$
\left|z \frac{f^{\prime}(z)}{f(z)}\right| \frac{1+\left.f(z)\right|^{2}}{1-|f(z)|^{2}} \leq \frac{1+|z|^{2}}{1-|z|^{2}}
$$


Since to every odd univalent function $f(z)$ there corresponds a function $\varphi(z) \in S$ and vice-versa such that $f(z)=\left[\varphi\left(z^{2}\right)\right]^{1 / 2}$, we get from (45) the following inequality for functions $\varphi(z) \in S$

$$
\left|z \frac{\varphi^{\prime}(z)}{\varphi(z)}\right| \frac{1+|\varphi(z)|}{1-|\varphi(z)|} \leq \frac{1+|z|}{1-|z|} .
$$

This was earlier obtained by Singh [12] by the method of interior variations.

Further, if $\varphi(z) \in S$, then for $|\zeta|<1$

$$
F(z)=\frac{\varphi\left(\frac{z+\zeta}{1+\bar{\zeta} z}\right)-\varphi(\zeta)}{1-\overline{\varphi(\zeta)} \varphi\left(\frac{z+\zeta}{1+\bar{\zeta} z}\right)}
$$

also belongs to $S$ and therefore satisfies (46). One readily concludes that

$$
-\zeta \frac{F^{\prime}(-\zeta)}{F(-\zeta)}=\frac{\zeta \varphi^{\prime}(0)\left[1-|\varphi(\zeta)|^{2}\right]}{\varphi(\zeta)\left(1-|\zeta|^{2}\right)}
$$

and $F(-\zeta)=-\varphi(\zeta)$. Hence (46) yields

$$
\frac{|\varphi(\zeta)|}{(1+|\varphi(\zeta)|)^{2}} \geq \frac{|\zeta| \varphi^{\prime}(0)}{(1+|\zeta|)^{2}}
$$

The inequalities in (46) and (49) are easily seen to be the best possible, equality being attained for the function

$$
\frac{\varphi(z)}{(1+\varphi(z))^{2}}=\frac{\varphi^{\prime}(0) z}{(1+z)^{2}} .
$$

If $\varrho$ is the distance from the origin of the nearest boundary point, then (49) gives

$$
\frac{\varrho}{(1+\varrho)^{2}} \geq \frac{1}{4} \varphi^{\prime}(0)
$$

which is a generalization of the Koebe quarter theorem.

In order to obtain the inequalities corresponding to (46) and (49) in the opposite direction, we set $z=r e^{i \Theta}$ in (46) and, for fixed $\Theta$, integrate with respect to $r$ from $r_{1}$ to $r_{2}$ and let $r_{1} \rightarrow 0$. We obtain

$$
\frac{|\varphi(z)|}{(1-|\varphi(z)|)^{2}} \leq \frac{\varphi^{\prime}(0)|z|}{(1-|z|)^{2}} .
$$


Using once again the transformation (47) we get

$$
\left|z \frac{\varphi^{\prime}(z)}{\varphi(z)}\right| \geq \frac{1-|z|}{1+|z|} \frac{1+|\varphi(z)|}{1-|\varphi(z)|} .
$$

The inequalities (52) and (53) are easily seen to be sharp, equality holding for the function

$$
\frac{\varphi(z)}{(1-\varphi(z))^{2}}=z \frac{\varphi^{\prime}(0)}{(1-z)^{2}} .
$$

We have therefore proved the following

Theorem 3. Let $S$ be the class of schlicht functions $f(z)$, in $|z|<1$, such that $f(0)=0, f^{\prime}(0)=a_{1}>0$ and $|f(z)|<1$. Then, for $|z|=\varrho<1$, the following sharp inequalities hold:

$$
\begin{aligned}
& \frac{2 a_{1} \varrho}{\left(1+2 \varrho\left(1-a_{1}\right)+\varrho^{2}\right)+(1+\varrho) \sqrt{1+2 \varrho\left(1-2 a_{1}\right)+\varrho^{2}}} \leq|f(z)| \\
& \leq \frac{2 a_{1} \varrho}{\left(1-2 \varrho\left(1-a_{1}\right)+\varrho^{2}\right)+(1-\varrho) \sqrt{1-2 \varrho\left(1-2 a_{1}\right)+\varrho^{2}}}
\end{aligned}
$$

and

$$
\frac{1-\varrho}{\left[1+2 \varrho\left(1-2 a_{1}\right)+\varrho^{2}\right]^{1 / 2}} \leq\left|z \frac{f^{\prime}(z)}{f(z)}\right| \leq \frac{1+\varrho}{\left[1-2 \varrho\left(1-2 a_{1}\right)+\varrho^{2}\right]^{1 / 2}}
$$

In each case, the boundary of the extremal domain consists of the unit circumference with a radial slit towards the center.

We mention that the inequalities (55) have been obtained from (49) and (52) by using the fact that the left hand sides of (49) and (52) involve monotonic functions of $|\varphi(z)|$ and hence they can be solved for $|\varphi(z)|$. Similarly, inequalities (56) have been obtained from (46) and (53) by substituting the corresponding extreme value for the quantity $(1+|\varphi(z)|) /(1-|\varphi(z)|)$. The inequalities (55) and (56) are easily seen to include, as special cases, the well-known distortion theorems for schlicht functions.

\section{$\S 3$. Coefficient Inequalities}

Let the domain $D$ be the unit disc and let us consider the class $S$ of bounded schlicht functions which have the expansion (1). Since in this case $k_{\mu \nu}=\frac{1}{\pi} \mu \delta_{\mu \nu}$ and $\chi_{\mu \nu}=0$, the inequality (40) becomes

$$
\left|\sum_{\mu, \nu=1}^{N} \alpha_{\mu} \alpha_{\nu} C_{\mu \nu}\right| \leq \sum_{\mu=1}^{N} \mu\left|\alpha_{\mu}\right|^{2}-\sum_{\mu, \nu=1}^{n} \alpha_{\mu} \bar{\alpha}_{\nu} d_{\mu \nu} .
$$


These inequalities are equivalent to the inequalities obtained by Nehari [6].

We observe that if $f(z) \in S$ then $F(z)=\left[f\left(z^{2}\right)\right]^{1 / 2}$ also belongs to $S$. It is readily verified that

$$
F(z)
$$

$=z \sqrt{b_{1}}\left\{1+\frac{z^{2}}{2} \frac{b_{2}}{b_{1}}+\frac{z^{4}}{2}\left(\frac{b_{3}}{b_{1}}-\frac{1}{4} \frac{b_{2}^{2}}{b_{1}^{2}}\right)+\frac{z^{6}}{2}\left(\frac{b_{4}}{b_{1}}-\frac{1}{2} \frac{b_{2} b_{3}}{b_{1}^{2}}+\frac{1}{16} \frac{b_{2}^{3}}{b_{1}^{3}}\right)+\ldots\right.$.

Taking $N=3$ and using (57) for the function $F(z)$ we obtain (59)

$$
\begin{aligned}
& \left|\frac{1}{2} \frac{b_{2}}{b_{1}} \alpha_{1}^{2}+\frac{1}{2}\left(\frac{b_{3}}{b_{1}}-\frac{b_{2}^{2}}{b_{1}^{2}}\right) \alpha_{2}^{2}+\left(\frac{b_{3}}{b_{1}}-\frac{3}{4} \frac{b_{2}^{2}}{b_{1}^{2}}\right) \alpha_{1} \alpha_{3}+\frac{\alpha_{3}^{2}}{2}\left(\frac{b_{4}}{b_{1}}-\frac{2 b_{2} b_{3}}{b_{1}^{2}}+\frac{13}{12} \frac{b_{2}^{3}}{b_{1}^{3}}\right)\right| \\
& \leq\left(1-b_{1}\right)\left|\alpha_{1}\right|^{2}+\left(1-b_{1}^{2}\right) \frac{\left|\alpha_{2}\right|^{2}}{2}+\left(1-b_{1}^{3}-\frac{3}{4} \frac{\left|b_{2}\right|^{2}}{b_{1}}\right) \frac{\left|\alpha_{3}\right|^{2}}{3}-b_{1} \operatorname{Re}\left\{\alpha_{1} \bar{\alpha}_{3} \bar{b}_{2} / b_{1}\right\} .
\end{aligned}
$$

Putting $\alpha_{2}=\alpha_{3}=0$ in (59) we get

$$
\left|b_{2}\right| \leq 2 b_{1}\left(1-b_{1}\right)
$$

an inequality due to Pick [7] and setting $\alpha_{1}=\alpha_{3}=0$ we have

$$
\left|\frac{b_{3}}{b_{1}}-\frac{b_{2}^{2}}{b_{1}^{2}}\right| \leq\left(1-b_{1}^{2}\right)
$$

which is the adaptation of Bieberbach area inequality to our present case. Both these inequalities are sharp, equality holding only for the functions given by (54), with $\varphi^{\prime}(0)=b_{1}$, and those obtained from it by rotation. We now proceed to prove the following

Theorem 4. Let $S_{1}$ denote the subclass of the class $S$ of bounded univalent functions $f(z)$ with expansion (1) such that the coefficients $b_{n}$ are real. The following sharp inequalities hold for the coefficient $b_{4}$ :

(i) For $4 / 9 \leq b_{1} \leq 1$,

$$
b_{4} \leq \frac{2}{3} b_{1}\left(1-b_{1}^{3}\right) \text {. }
$$

Equality is attained only for the function $f(z)$ given by

$$
\frac{f^{3}(z)}{\left(1-f^{3}(z)\right)^{2}}=\frac{b_{1}^{3} z^{3}}{\left(1-z^{3}\right)^{2}}
$$

The boundary of the extremal domain consists of the unit circumference together with three radial slits of equal length starting at the points $e^{ \pm \pi i / 3}$ and -1 .

(ii) For $1 / 11 \leq b_{1} \leq 4 / 9$ 


$$
b_{4} \leq \frac{2}{3} b_{1}\left(1-b_{1}^{3}\right)+\frac{8}{49} b_{1}\left(4-9 b_{1}\right)^{3}
$$

and the corresponding extremum function is given by

$$
\frac{f^{3}(z)}{\left[f^{3}(z)+\frac{3 \lambda}{b_{1}} f^{2}(z)-\frac{3 \lambda}{b_{1}} f(z)-1\right]^{2}}=\frac{b_{1}^{3} z^{3}}{\left(z^{3}+\lambda z^{2}-\lambda z-1\right)^{2}}
$$

where $\lambda=\frac{6}{7}\left(4-9 b_{1}\right)=\frac{3}{2}(1-2 \cos \varphi)=b_{1}(1-2 \cos \Theta)$.

The boundary of the extremal domain consists of the unit circumference together with a straight slit at the point -1 and two slits starting at the points $e^{ \pm i \Theta}$ and symmetric with respect to the real axis. The end points of the slits are images, respectively, of the points $-1, e^{ \pm i \varphi}$.

(iii) For $0 \leq b_{1} \leq 1 / 11$,

$$
b_{4} \leq \frac{2}{3} b_{1}\left(1-b_{1}^{3}\right)+\frac{10 b_{1}}{3}\left(1-b_{1}\right)^{2}\left(1-4 b_{1}\right) \text {. }
$$

There is strict inequality unless

$$
\frac{f(z)}{(1-f(z))^{2}}=\frac{b_{1} z}{(1-z)^{2}}
$$

and the extremal boundary consists of the unit circumference, together with a straight slit starting at the point -1 .

Proof. In (59) take $\alpha_{2}=0$ and put $\alpha_{1} / \alpha_{3}=\chi$ real. We then obtain

$$
\begin{aligned}
\left(\frac{b_{2}}{b_{1}} \chi^{2}\right. & +2 \chi\left(\frac{b_{3}}{b_{1}}-\frac{3}{4} \frac{b_{2}^{2}}{b_{1}^{2}}\right)+\left(\frac{b_{4}}{b_{1}}-\frac{2 b_{2} b_{3}}{b_{1}^{2}}+\frac{13}{12} \frac{b_{2}^{3}}{b_{1}^{3}}\right) \\
& \leq 2\left(1-b_{1}\right) \chi^{2}-2 \chi b_{2}+\frac{2}{3}\left(1-b_{1}^{3}-\frac{3}{4} \frac{b_{2}^{2}}{b_{1}}\right) .
\end{aligned}
$$

Since we can assume $b_{4}>0$ we get, on taking the real part of the left hand side,

$$
\begin{aligned}
\frac{b_{4}}{b_{1}} \leq \frac{2 b_{2} b_{3}}{b_{1}^{2}} & -\frac{13}{12} \frac{b_{2}^{3}}{b_{1}^{3}}+\frac{2}{3}\left(1-b_{1}^{3}-\frac{3}{4} \frac{b_{2}^{2}}{b_{1}}\right) \\
& -2 \chi\left(b_{2}+\frac{b_{3}}{b_{1}}-\frac{3}{4} \frac{b_{2}^{2}}{b_{1}^{2}}\right)+2 \chi^{2}\left(1-b_{1}-\frac{b_{2}}{2 b_{1}}\right)
\end{aligned}
$$


We choose $\chi$ so that it minimizes the right hand side. This value is given by

$$
\chi=\frac{\left[b_{2}+b_{3} / b_{1}-\frac{3}{4} b_{2}^{2} / b_{1}^{2}\right]}{2\left[1-b_{1}-b_{2} / 2 b_{1}\right]}
$$

and (69) now yields

$$
\begin{aligned}
\frac{b_{4}}{b_{1}} \leq \frac{2 b_{2}}{b_{1}}\left(b_{2}+\frac{b_{3}}{b_{1}}-\frac{3}{4} \frac{b_{2}^{2}}{b_{1}^{2}}\right)- & \frac{\left(b_{2}+\frac{b_{3}}{b_{1}}-\frac{3}{4} \frac{b_{2}^{2}}{b_{1}^{2}}\right)^{2}}{2\left(1-b_{1}-b_{2} / 2 b_{1}\right)} \\
& +\frac{2}{3}\left(1-b_{1}^{3}\right)+\frac{5}{12} \frac{b_{2}^{3}}{b_{1}^{3}}-\frac{5 b_{2}^{2}}{b_{1}} .
\end{aligned}
$$

But

$$
\frac{2 b_{2}}{b_{1}}\left(b_{2}+\frac{b_{3}}{b_{1}}-\frac{3}{4} \frac{b_{2}^{2}}{b_{1}^{2}}\right)-\frac{\left(b_{2}+\frac{b_{3}}{b_{1}}-\frac{3}{4} \frac{b_{2}^{2}}{b_{1}^{2}}\right)^{2}}{2\left(1-b_{1}-b_{2} / 2 b_{1}\right)} \leq \frac{2 b_{2}^{2}}{b_{1}^{2}}\left(1-b_{1}-\frac{b_{2}}{2 b_{1}}\right)
$$

equality holding only when

$$
b_{2}+\frac{b_{3}}{b_{1}}-\frac{3}{4} \frac{b_{2}^{2}}{b_{1}^{2}}=2\left(1-b_{1}-\frac{b_{2}}{2 b_{1}}\right) \frac{b_{2}}{b_{1}} .
$$

Using (71) we obtain from (70)

$$
\frac{b_{4}}{b_{1}} \leq \frac{2}{3}\left(1-b_{1}^{3}\right)+\frac{1}{2} \frac{b_{2}^{2}}{b_{1}^{2}}\left(4-9 b_{1}\right)-\frac{7}{12} \frac{b_{2}^{3}}{b_{1}^{3}} .
$$

One easily confirms that if $1 \geq b_{1} \geq 4 / 9$, the maximum of the right hand side of (73) is attained for $b_{2}=0$. Hence

$$
\frac{b_{4}}{b_{1}} \leq \frac{2}{3}\left(1-b_{1}^{3}\right), \quad 1 \geq b_{1} \geq 4 / 9,
$$

equality holding only when $b_{2}=0$ and hence from $(72) b_{3}=0$. The extremal function is easily seen to be

$$
\frac{f^{3}(z)}{\left(1-f^{3}(z)\right)^{2}}=\frac{b_{1}^{3} z^{3}}{\left(1-z^{3}\right)^{2}} .
$$

This proves part (i) of the theorem.

We need to remark now that $b_{2}>0$ for the function for which $b_{4}$ is maximum. This is a consequence of the well-knovn inequality $\left|b_{n+1}-b_{n-1}\right| \leq 2 b_{1}$ for symmetric univalent functions. Indeed, if $b_{2}$ were 
A. I. 310

not positive, it would follow that $b_{4} \leq 2 b_{1}$ but the functions given by (65) and (67) yield values of $b_{4} / b_{1}$ which are greater than 2 .

Let us now assume $0 \leq b_{1} \leq 4 / 9$ and maximize the right hand side of (73) as a function of $b_{2} / b_{1}$. This is seen to occur for

$$
7 b_{2}=4 b_{1}\left(4-9 b_{1}\right)
$$

and the corresponding inequality for $b_{4}$ is

$$
\frac{b_{4}}{b_{1}} \leq \frac{2}{3}\left(1-b_{1}^{3}\right)+\frac{8}{49}\left(4-9 b_{1}\right)^{3} .
$$

Equality holds in (77) for the function for which $b_{2}$ is given by (76) and by virtue of (72)

$$
\frac{b_{3}}{b_{1}}=\frac{8}{49}\left(4-9 b_{1}\right)\left(5-6 b_{1}\right),
$$

and it is easily confirmed that the function given by (65) gives equality in (77) and satisfies (76) and (78).

In view of the inequality (60) one finds that (76) and hence (77) hold for $1 / 11 \leq b_{1} \leq 4 / 9$. At $b_{1}=4 / 9$ the extremal values given by $(74)$ and (77) agree. This proves part (ii) of the theorem.

We now have $0 \leq b_{1} \leq 1 / 11$. Let us set

$$
\frac{b_{2}}{b_{1}}=2 \lambda\left(1-b_{1}\right), \quad 0 \leq \lambda \leq 1 \text {. }
$$

In view of $(60)$ we find that the entire region of variation of positive values of $b_{2}$ is covered by straight lines of the system (79) such that through each point there passes only one line of the family. Substituting in (73), this value of $b_{2} / b_{1}$, we obtain

$$
\frac{b_{4}}{b_{1}} \leq \frac{2}{3}\left(1-b_{1}^{3}\right)+2 \lambda^{2}\left(1-b_{1}\right)^{2}\left(4-9 b_{1}\right)-\frac{14}{3} \lambda^{3}\left(1-b_{1}\right)^{3} .
$$

We note that the right hand side of $(80)$ is a monotone increasing function of $\lambda$ for $0 \leq b_{1} \leq 1 / 11$. Indeed, its derivative with respect to $\lambda$ is easily seen to be $2\left(1-b_{1}\right)^{2} \lambda\left[(8-7 \lambda)+b_{1}(7 \lambda-18)\right]$ which is positive if $b_{1} \leq(8-7 \lambda) /(18-7 \lambda)$. Since $0 \leq \lambda \leq 1$, this is seen to hold for $0 \leq b_{1}$ $\leq 1 / 11$. Hence the maximum in (80) is attained for $\lambda=1$. The corresponding inequality for $b_{4}$ is

$$
\frac{b_{4}}{b_{1}} \leq \frac{2}{3}\left(1-b_{1}^{3}\right)+\frac{10}{3}\left(1-b_{1}\right)^{2}\left(1-4 b_{1}\right)
$$


where equality holds only when $b_{2} / b_{1}=2\left(1-b_{1}\right)$. Hence the last part of the theorem follows from the fact that equality in (60) is attained only for the function

$$
\frac{f(z)}{(1-f(z))^{2}}=\frac{b_{1} z}{(1-z)^{2}} .
$$

This completes the proof.

It should be remarked here that equality statements in (62) and (64) and the fact that the corresponding functions (63) and (65) are the only extremal functions are not easy to deduce from Grunsky inequality. These statements, however, are immediately verified if one uses the method of interior variations [12] because the differential equation pertaining to the cases (i) and (ii) above can then be integrated in a closed form. Further, the restriction that $b_{n}$ be real is purely artificial and the theorem should hold for the class $S$ but the present technique does not yield this strong form.

Let us now consider the class $\Sigma$ of functions $f(z)$ univalent in $|z|>1$ with $|f(z)|>1$ for $|z|>1$ and having the following expansion at $W$

$$
f(z)=c z+\frac{c_{1}}{z}+\frac{c_{2}}{z^{2}}+\frac{c_{3}}{z^{3}}+\ldots, \quad c>1 .
$$

It is easily verified that the inequalities (43) hold if $f(z) \in \Sigma$ with the difference that $|\zeta|>1$. Let $F_{m}(t)$ denote the polynomial of degree $m$ in $t$ such that

$$
F_{m}(f(z))=z^{m}+\sum_{n=1}^{\infty} a_{m n} z^{-n}
$$

and let us put

$$
F_{m}\left(\frac{1}{\overline{f(\zeta)}}\right)=\sum_{n=1}^{\infty} \frac{b_{m n}}{\overline{\zeta^{n}}}
$$

$F_{m}(t)$ is in fact the Faber polynomial [10] of degree $m$ for $f(z)$.

By expanding the functions in (43) about the point at $\infty$ one obtains the following analogue of the inequalities (57)

$$
\left|\sum_{\mu, v=1}^{N} v \alpha_{\mu} \alpha_{\nu} \alpha_{\mu \nu}\right|=\sum_{\mu=1}^{N} \mu\left|\alpha_{\mu}\right|^{2}-\sum_{\nu, \mu=1}^{N} v \alpha_{\mu} \bar{\alpha}_{\nu} b_{\mu \nu}, \quad N=1,2,3, \ldots
$$

Taking $\mathrm{N}=2$ and putting $\alpha_{2}=0$ we get

$$
\left|c_{1}\right| \leq c\left(1-\frac{1}{c^{2}}\right)
$$

and on setting $\alpha_{1}=0$ we have

$$
\left|2 \frac{c_{3}}{c}+\frac{c_{1}^{2}}{c^{2}}\right| \leq 1-\frac{1}{c^{4}} .
$$


A. I. 310

Equations (19) show that equality holds in (84) and (85) only for the domain bounded by the unit circumference with a rectilinear slit in the direction away from the center.

Let us apply the inequality (83) to the function

$$
F(z)=\left[f\left(z^{2}\right)\right]^{1 / 2}=z \sqrt{c}+\frac{1}{2} \frac{c_{1}}{\sqrt{c} z^{3}}+\frac{1}{2} \frac{c_{2}}{\sqrt{c} z^{5}}+\ldots
$$

which also belongs to $\Sigma$ if $f(z) \in \Sigma$. On taking $N=3$ in (83) and setting $\alpha_{1}=\alpha_{2}=0$ we obtain

$$
\left|\frac{c_{2}}{c}\right| \leq \frac{2}{3}\left(1-\frac{1}{c^{3}}\right)
$$

The extremal domain in (86) is the domain bounded by the unit circumference with three symmetrically situated rectilinear slits of equal length. In the limit when $c \rightarrow \infty$ this yields an inequality due to Schiffer [8] and Golusin [4] for the class $\Sigma_{1}$.

Thus we have proved the following

Theorem 5. Let $f(z) \in \Sigma$ have the expansion (2). Then the coefficients $c_{1}$ and $c_{2}$ satisfy the inequalities (84) and (86). There is strict inequality in (84) unless the domain is bounded by the unit circumference with a radial slit away from the center. In (86) equality is attained only for the domain bounded by the unit circumference with three symmetrically situated radial slits, of equal length, in the direction away from the origin.

Finally, let us consider the class $\Sigma^{\prime}$ of functions which are inverse of the functions belonging to $\Sigma$. It is seen without any difficulty that if $g(w) \in \Sigma^{\prime}$ and near infinity

$$
g(w)=d w+\frac{d_{1}}{w}+\frac{d_{2}}{w^{2}}+\frac{d_{3}}{w^{3}}+\ldots, \quad 1 \leq d<0,
$$

then if $g(w)$ is the inverse of $f(z) \in \Sigma$ with expansion (3), we have

$$
d=\frac{1}{c}, d_{1}=-c_{1}, \quad d_{2}=-c_{2} c, \text { and } \frac{d_{3}}{c^{3}}=-\left(\frac{c_{3}}{c}+\frac{c_{1}^{2}}{c^{2}}\right)
$$

Hence (84) yields

$$
\left|d_{1}\right| \leq \frac{1}{d}\left(1-d^{2}\right)
$$

and (86) provides

$$
\left|d_{2}\right| \leq \frac{2}{3 d^{2}}\left(1-d^{3}\right)
$$


Further, (85) gives

$$
\left|d_{3}\right| \leq \frac{1}{d^{3}}\left(1-d^{2}\right)
$$

The last inequality is a generalization of the corresponding inequality due to Springer [13].

We have, therefore, proved the following.

Theorem 6. Let the functions of the class $\Sigma^{\prime}$ have the expansion (4). Then the coefficients $d_{1}, d_{2}$, and $d_{3}$ satisfy respectively the inequalities (87), (88), and (89). Equality in (87) and (89) holds for the domains bounded by the unit circumference with a rectilinear slit pointing away from the center. Equality in (88) holds for the domain bounded by the unit circumference with three symmetrically rectilinear slits of equal length in the direction away from the origin.

Harvard University

Cambridge, Mass., U.S.A.

and

D. A. V. College

Kanpur, India 


\section{References}

[1] Bergman, S.: The kernel function and conformal mapping. - Mathematical Surveys V, American Mathematical Society, New York, 1950.

[2] -》- and Schiffer, M.: Kernel functions and conformal mapping. - Compositio Math. 8 (1951), 205-249.

[3] Charzynski, Z. and Schiffer, M.: A new proof of the Bieberbach conjecture of the fourth coefficient. - Arch. Rational Mech. Anal. 5 (1960), 187-193.

[4] Golusin, G. M.: Einige Koeffizientenabschätzungen für schlichte Funktionen. Rec. Math. Moscou, N. S. 3 (1938), 321-330, Russian with a German summary.

[5] Grunsky, H.: Koeffizientenbedingungen für schlicht abbildende meromorphe Funktionen. - Math. Z. 45 (1939), 29-61.

[6] Nehari, Z.: Some inequalities in the theory of functions. - Trans. Amer. Math. Soc. 75 (1953), $256-286$.

[7] PICk, G.: Über die konforme Abbildung eines Kreises auf ein schlichtes und zugleich beschränktes Gebiet. - S.-B. Math. Nat. Kl. Kaiserl. Akad. Wiss. Wien, 2. Abt. 126 (1917), 247-263.

[8] Schiffer, M.: Sur un problème d'extrémum de la représentation conforme. Bull. Soc. Math. France 66 (1938), 48-55.

[9] - - The kernel function of an orthonormal system. - Duke Math. J. 13 (1946), $529-540$.

[10] -》- Faber polynomials in the theory of univalent functions. - Bull. Amer. Math. Soc. 54 (1948), 503-557.

[11] - - and Spencer, D. C.: On the conformal mapping of one Riemann surface into another. - Ann. Acad. Scient. Fennicæ A I 94 (1951).

[12] Singh, V.: Interior variations and some extremal problems for certain classes of univalent functions. - Pacific J. Math. 7 (1957), 1485-1504.

[13] Springer, G.: The coefficient problem for schlicht mappings of the exterior of the unit circle. - Trans. Amer. Math. Soc. 70 (1951), 421-450. 